International Journal of Pure and Applied Mathematics

Volume 110 No. 4 2016, 651-656

ISSN: 1311-8080 (printed version); ISSN: 1314-3395 (on-line version)

url: http://www.ijpam.eu

doi: 10.12732/ijpam.v110i4.7

\title{
ORBIT OF TUPLE OF OPERATORS TENDING TO INFINITY
}

\author{
Abdelaziz Tajmouati $^{1 \S}$, Youness Zahouan ${ }^{2}$ \\ ${ }^{1,2}$ Laboratory of Mathematical Analysis and Applications \\ Faculty of Sciences Dhar Al Mahraz \\ Sidi Mohammed Ben Abdellah University \\ Fez, MOROCCO
}

\begin{abstract}
In this paper we prove that there is a dense set of vectors in $X$ whose orbits under the tuple $\mathcal{T}=\left(T_{1}, T_{2}, \ldots, T_{n}\right)$ of commutative bounded linear operators on a infinite dimensional (real, complex) Banach space $X$ tend to infinity.
\end{abstract}

Key Words: tuple of operators, orbit, spectral radius, spectrum, point spectrum, approximate point spectrum, bounded below operator

\section{Introduction}

By an $n$-tuple of operators we mean a finite sequence of length $n$ of commuting bounded linear operators on a Banach space $X$.

Throughout, $X$ denotes a infinite dimensional Banach space and $B(X)$ denotes the Banach algebra of all bounded linear operators on $X$. The orbit of a points $x \in X$ under an operator $T \in B(X)$ is the sequence

$$
\left\{T^{n} x: n=0,1, \ldots\right\} .
$$

For $T \in B(X)$, with $\mathrm{r}(\mathrm{T}), \sigma(T), \sigma_{p}(T)$ and $\sigma_{a p}(T)$ will denote the spectral radius, the spectrum, the point spectrum and the approximate point spectrum of T,respectively. Recall that $\sigma_{p}(T)$ is the set of all eigenvalues of $\mathrm{T}$ while $\sigma_{a p}(T)$ is the set of $\lambda \in \sigma(T)$ for there which there is a sequence of unit vectors

Received: $\quad$ August 17, 2016

Revised: $\quad$ October 4, 2016

Published: November 9, 2016

$\S_{\text {Correspondence author }}$ (c) 2016 Academic Publications, Ltd.

url: www.acadpubl.eu 
$\left(x_{n}\right)_{n \geq 1}$ such that $\left\|T x_{n}-\lambda x_{n}\right\| \rightarrow 0$, as $n \rightarrow \infty$ any such sequence is called a sequence of almost eigenvectors for $\lambda$.

We are interested in the operators $T \in B(X)$ for which there is $x \in X$ whose $\operatorname{Orb}(T, x)$ tends strongly to infinity, i.e.

$$
\left\|T^{n} x\right\| \longrightarrow \infty \text {, as } n \longrightarrow \infty \text {. }
$$

Obviously, if $\sigma_{p}(T)$ contains a point $\lambda$ with $|\lambda|>1$, then for every corresponding nonzero vector $x$ in the eigenspace $\operatorname{Ker}(T-\lambda)$, the orbit will tends strongly to infinity:

$$
\left\|T^{n} x\right\|=\left|\lambda^{n}\right|\|x\| \longrightarrow \infty, \text { as } n \longrightarrow \infty .
$$

In general, $\operatorname{Ker}(T-\lambda)$ is not dense in $\mathrm{X}$ (relative to the norm topology). In order to produce a dense set of vectors in $X$ whose orbits under $T$ tend strongly to infinity we have to look at the points in the approximate point spectrum which are not eigenvalues.

In [5] S. Mancevska gaves a complete proof of the following theorem (originally stated by B. Beauzamy [9, Theorem 2.A.5]): If $T \in B(X)$ and the circle $\{\lambda \in \mathbb{C} /|\lambda|=r(T)\}$ contains a point in $\sigma(T)$ which is not an eigenvalue for $\mathrm{T}$, then for every positive sequence $\left(\alpha_{n}\right)_{n \geq 1}$ with $\sum_{n \geq 1} \alpha_{n}<+\infty$, in every open ball in $\mathrm{X}$ with radius strictly larger then $\sum_{n \geq 1} \alpha_{n}$, there is $x \in X$ satisfying $\left\|T^{n} x\right\| \geq \alpha_{n} \frac{r(T)^{n}}{2}$ for all $n \geq 1$. Note that, if $r(T)>1$, then the space will contain a dense set of vectors $x \in X$ with orbits under $T$ tending strongly to infinity.

If $r(T)$ is replaced with $|\lambda|$, for any $\lambda \in \sigma_{a p}(T) \backslash \sigma_{p}(T)$. Thus we have.

Theorem 1.2. (see [1], Corollary 3.2.) Let $X$ be an infinite dimensional reflexive Banach space and $T \in B(X)$ and $S \in B(X)$.

If the sets $\sigma_{a p}(T) \backslash \sigma_{p}(T)$ and $\sigma_{a p}(S) \backslash \sigma_{p}(S)$ both have a non-empty intersection with the domain $\{\lambda \in \mathbb{C} /|\lambda|>1\}$ then, there is a dense set of vectors $x \in X$ such that both the orbits $\operatorname{Orb}(T ; x)$ and $\operatorname{Orb}(S ; \mathrm{x})$ tend strongly to infinity.

In [2] S. Mancevska and M. Orovrance considered some conditions under which, given a sequence of bounded linear operators $\left(T_{i}\right)_{i \geq 1}$ on an infinitedimensional complex reflexive Banach space $\mathrm{X}$, and they show that there is a dense set of vectors in $X$ whose orbits under each $T_{i}$ tend strongly to infinity.

Theorem 1.3. (see [2], Corollary 10) Let $X$ an infinite dimensional reflexive Banach space. If $\left(T_{i}\right)_{i \geq 1}$ is a sequence in $B(X)$ for wich there is $\beta>0$ such that $r\left(T_{i}\right)>1+\beta$ for all $i \geq 1$ then, there is a dense set $D$ in $X$ such that $\operatorname{Orb}\left(T_{i} ; x\right)$ tend strongly to infinity for every $x \in D$ and $i \geq 1$. 
If $\left(T_{i}\right)_{i \geq 1}$ is a sequence in $B(X)$ satisfying the following, weaker condition then the one in Theorem 0.3.

$$
\sigma_{a p}\left(T_{i}\right) \backslash \sigma_{a}\left(T_{i}\right) \cap\{\lambda \in \mathbb{C} /|\lambda| \succ 1\} \neq \emptyset, \quad \text { for all } i \geq 1
$$

the space may still contain a dense set of vectors with orbits under each $T_{i}$, $i \geq 1$ tending strongly to infinity.

\section{Main Results}

Definition 1.1. Let $\mathcal{T}=\left(T_{1}, T_{2}, \ldots, T_{n}\right)$ be an $n$-tuple of operators acting on an infinite dimensional Banach space $\mathrm{X}$.

Let

$$
\mathcal{F}=\left\{T_{1}^{k_{1}} T_{2}^{k_{2}} \ldots T_{n}^{k_{n}}: k_{i} \geq 0, i=1, \ldots, n\right\} .
$$

be the semi-group generated by $\mathcal{T}$. For $x \in X$, the orbit of $\mathrm{x}$ under the tuple $\mathcal{T}$ is the set

$$
\operatorname{Orb}(\mathcal{T}, x)=\{S x: S \in \mathcal{F}\}
$$

Definition 1.2. The orbit of $\mathrm{x}$ under the tuple $\mathcal{T}$ tending to infinity if:

$$
\left\|T_{1}^{k_{1}} T_{2}^{k_{2}} \ldots T_{n}^{k_{n}} x\right\| \rightarrow \infty
$$

as $k_{i} \rightarrow \infty$ with $k_{i} \geq 0$, for all $i=1, \ldots, n$.

In this paper are considered some conditions under which, The orbit of $\mathrm{x}$ under the tuple $\mathcal{T}$ tending to infinity. For simplicity we state and prove our results for a pair that is a tuple with $n=2$, and the general case follows by a similar method.

Definition 1.3. An operator $T$ is bounded from below if and only there exists a constant $C>0$ such that:

$$
\|T x\| \geq C\|x\|, \text { for all } x \in X
$$

Theorem 1.4. Let $X$ an infinite dimensional reflexive Banach space and $T=\left(T_{1}, T_{2}\right)$ be the pair of operators $T_{1}$ and $T_{2}$.

Suppose that the fllowing conditions hold true:

1) $T_{1}$ and $T_{2}$ are bounded from below. 
2) The sets $\sigma_{a p}(T) \backslash \sigma_{p}(T)$ and $\sigma_{a p}(S) \backslash \sigma_{p}(S)$ both have a non-empty intersection with the domain $\{\lambda \in \mathbb{C} /|\lambda|>1\}$.

Then there exists $x \in X$ such that the orbit of $x$ under the pair $T$ tends strongly to infinity.

Proof. Using condition 2 we may apply Theorem 1.2. Then, there exists a dense set of vectors $x \in X$ such that both the orbits $\operatorname{Orb}\left(T_{1} ; x\right)$ and $\operatorname{Orb}\left(T_{2} ; x\right)$ tend strongly to infinity.

Hence $T_{1}$ and $T_{2}$ are bounded from below or,

$$
\begin{aligned}
\left\|T_{1}^{k_{1}} T_{2}^{k_{2}} x\right\| & =\left\|T_{1}^{k_{1}}\left(T_{2}^{k_{2}} x\right)\right\| \\
& \geq C_{1}\left\|T_{2}^{k_{2}} x\right\| \rightarrow \infty, \text { as } k_{2} \rightarrow \infty,
\end{aligned}
$$

and

$$
\begin{aligned}
\left\|T_{1}^{k_{1}} T_{2}^{k_{2}} x\right\| & =\left\|T_{2}^{k_{2}} T_{1}^{k_{1}} x\right\| \\
& =\left\|T_{2}^{k_{2}}\left(T_{1}^{k_{1}} x\right)\right\| \\
& \geq C_{2}\left\|T_{1}^{k_{1}} x\right\| \rightarrow \infty, \text { as } k_{1} \rightarrow \infty .
\end{aligned}
$$

Then

$$
\left\|T_{1}^{k_{1}} T_{2}^{k_{2}} x\right\| \rightarrow \infty \text { as } k_{1} \rightarrow \infty, \text { and } k_{1} \rightarrow \infty
$$

i.e. $\operatorname{Orb}(T, x)$ tend strongly to infinity.

Corollary 1.1. Let $X$ an infinite dimensional reflexive Banach space and $\mathcal{T}=\left(T_{1}, T_{2}, \ldots, T_{n}\right)$ be the $n$-tuple of operators in $B(X)$ bounded below for all $i \geq 1$.

If there is $x \in X$ such that the orbit of $\mathrm{x}$ under $T_{i}$ for all $i \geq 1$ tend strongly to infinity then the orbit of $\mathrm{x}$ under the tuple $\mathcal{T}$ tend strongly to infinity.

Remark 1.1. The converse is also true, ie If there is $x \in X$ such that the orbit of $\mathrm{x}$ under the tuple $\mathcal{T}$ tend strongly to infinity then the orbit of $\mathrm{x}$ under $T_{i}$ for all $i \geq 1$ tend strongly to infinity.

Proof. $T_{i}$ are the commuting bounded linear operators then,

$$
\left\|T_{1}^{k_{1}} T_{2}^{k_{2}} \ldots T_{n}^{k_{n}} x\right\| \leq \prod_{i=1, i \epsilon_{-j}}^{n}\left\|T_{i}^{k_{i}}\right\|\left\|T_{j}^{k_{j}} x\right\|,
$$

or

$$
\left\|T_{1}^{k_{1}} T_{2}^{k_{2}} \ldots T_{n}^{k_{n}} x\right\| \rightarrow \infty \text { as } k_{j} \rightarrow \infty \text { for all } j \geq 1
$$


Therefore, $\operatorname{Orb}\left(T_{j}, x\right) \rightarrow \infty$ for all $j \geq 1$.

Example 1.1. Let $S$ be the unilateral forward shift on $\ell^{2}(\mathbb{N})$ :

$$
S e_{n}=e_{n+1}, \quad i \geq 1
$$

where $\left\{e_{n}: n \in \mathbb{N}\right\}$ is the standard orthonormal basis for $\ell^{2}(\mathbb{N})$.

Given a sequence of positive numbers $\left(a_{i}\right)_{i \geq 1}$ so that $a_{i} \succ 1$ for all $i \geq 1$ and $a_{i} \rightarrow 1$ as $i \rightarrow \infty$ and let

$$
T_{i}=a_{i} S, \quad i=1, \ldots, n
$$

$T_{i}$ is unilateral injective forward weighted shift and hence (see [10], Theorem $6)$ :

$$
\sigma_{p}\left(T_{i}\right)=\emptyset \text { and } \sigma_{a p}\left(T_{i}\right)=\left\{\lambda \in \mathbb{C} /|\lambda|=a_{i}\right\}
$$

Then the set $\sigma_{a p}\left(T_{i}\right) \backslash \sigma_{p}\left(T_{i}\right)$ have a non-empty intersection with the domain $\{\lambda \in \mathbb{C} /|\lambda| \succ 1\}$.

Obviously, $\left(T_{i}\right)_{i \geq 1}$ satisfies the weaker condition $\left(^{*}\right)$ and there exists a dense set of vectors in $\ell^{2}(\mathbb{N})$ with orbits in each $T_{i}$ tending strongly to infinity.

Actually

$$
\left\|T_{i}^{n} x\right\|=\left\|\left(a_{i} S\right)^{n} x\right\|=a_{i}^{n}\|x\| \rightarrow \infty, n \rightarrow \infty \text {, for all } x \neq 0 \text { and } i \geq 1 .
$$

Moreover, $T_{i}$ is bounded from below. Indeed:

$$
\left\|T_{i} x\right\|=\left\|a_{i} S x\right\| \geq\|S x\|=\|x\| .
$$

Therefore, by the use of Corollary 1.1, the orbit of $\mathrm{x}$ in the tuple $\left(T_{1}, T_{2}, \ldots\right.$, $T_{3}$ ) tends strongly to infinity.

\section{References}

[1] S. Mancevska, M. Orovcanec, Orbits tending srongly to infinity under pairs of operators on reflexive Banach spaces, Glasnik Matematicki, 43, No. 63 (2008), 195-204.

[2] S. Mancevskaw, M. Orovcanec, orbits tending to infinity under sequences of operators on Banach spaces, Glasnik Matematicki, 47, No. 2 (2008), 175-183.

[3] S. Mancevska, On orbits for pairs of operators on an infinite-dimensional complex Hilbert space, Kragujevac J. Math., 30 (2007), 293-304.

[4] S. Mancevska, M. Orovcanec, Orbits tending to infinity under sequences of operators on Hilbert spaces, Filomat, 21, No. 2 (2007), 161-171.

[5] V. Muller, J. Vrsovsky, Orbits of linear operators tending to infinity, Rocky Mountain J. Math., 39, No. 1 (2009), 219-230. 
[6] V.Muller, Weak orbits and a local capacity of operators, Int. Eq. Oper. Theory, 41 (2001), 230-253.

[7] N.S. Feldman, Hypercyclic tuples of operators and somewhere dense orbits, J. Math. Appl., 346 (2008), 82-98.

[8] B. Yousefi, Gh.R. Moghimi, On the hypercyclicity for a tuple of operator, 76, No. 2 (2012), 261-265

[9] B. Beauzamy, Introduction to Operator Theory and Invariant Subspaces, North Holland, 1988

[10] A.L. Shields, Weighted shift operators and analytic function theory, In: Topics in Operator Theory, Mathematical Surveys, No. 13 (Ed. C. Pearsy), 49-128, American Math. Soc., Providence, Rhode Island, 1974. 